\title{
Recognition Algorithm of Acoustic Emission Signals Based on Conditional Random Field Model in Storage Tank Floor Inspection Using Inner Detector
}

\author{
Yibo Li, ${ }^{1}$ Yuxiang Zhang, ${ }^{1}$ Huiyu Zhu, ${ }^{1}$ Rongxin Yan, ${ }^{2}$ Yuanyuan Liu, \\ Liying Sun, ${ }^{3}$ and Zhoumo Zeng ${ }^{1}$ \\ ${ }^{1}$ State Key Laboratory of Precision Measurement Technology and Instrument, Tianjin University, Tianjin 300072, China \\ ${ }^{2}$ Beijing Institute of Spacecraft Environment Engineering, Beijing 100094, China \\ ${ }^{3}$ School of Control and Mechanical Engineering, Tianjin Chengjian University, Tianjin 300384, China
}

Correspondence should be addressed to Yuxiang Zhang; zhangyx@tju.edu.cn

Received 19 March 2015; Revised 3 July 2015; Accepted 13 October 2015

Academic Editor: Marco Alfano

Copyright (C) 2015 Yibo Li et al. This is an open access article distributed under the Creative Commons Attribution License, which permits unrestricted use, distribution, and reproduction in any medium, provided the original work is properly cited.

\begin{abstract}
Acoustic emission (AE) technique is often used to detect inaccessible area of large storage tank floor with AE sensors placed outside the tank. For tanks with fixed roofs, the drop-back signals caused by condensation mix with corrosion signals from the tank floor and interfere with the online AE inspection. The drop-back signals are very difficult to filter out using conventional methods. To solve this problem, a novel AE inner detector, which works inside the storage tank, is adopted and a pattern recognition algorithm based on CRF (Conditional Random Field) model is presented. The algorithm is applied to differentiate the corrosion signals from interference signals, especially drop-back signals caused by condensation. Q235 steel corrosion signals and drop-signals were collected both in laboratory and in field site, and seven typical AE features based on hits and frequency are extracted and selected by mRMR (Minimum Redundancy Maximum Relevance) for pattern recognition. To validate the effectiveness of the proposed algorithm, the recognition result of CRF model was compared with BP (Back Propagation), SVM (Support Vector Machine), and HMM (Hidden Markov Model). The results show that training speed, accuracy, and ROC (Receiver Operating Characteristic) results of the CRF model outperform other methods.
\end{abstract}

\section{Introduction}

Acoustic emission (AE) is a beneficial method to test the corrosion of the floor without opening the storage tank [1-5]. In conventional online tank floor tests [6], sensors are fixed by magnets outside the tank wall to collect signals. However, the $\mathrm{AE}$ test is susceptible to outside intervention, such as sand collision and external vibration. To solve this problem, a newly invented $\mathrm{AE}$ detection equipment is adopted in tank floor inspection and it could work inside the tank to collect the AE signals to avoid external disturbance [7]. Meanwhile, the condition of acoustic field inside storage tank is complicated. The characteristics of many noise signals inside tank are quite similar to the corrosion signals of tank floor, which would seriously influence the result of the evaluation of tank floor.
For those tanks with fixed-roofs, warm gas in the tank condenses to droplets when it meets the cold roof. The droplets fall down from the roof to the water/oil surface and generate interference $\mathrm{AE}$ signals [8]. The interference signals caused by the droplets should be filtered out to secure the accuracy of corrosion source location and the efficiency of the tank floor evaluation. Guard sensors are usually employed with the aim of shielding droplets noise signals during $\mathrm{AE}$ test for tank bottom. However, the space inside the inner AE detector is small and the hardware system of guard sensors is complicated. So it is not suitable for inner AE detector to use guard sensors. For this reason, a specific pattern recognition algorithm is proposed to filter out the interference signals.

Pattern recognition is often applied to identify AE signals caused by different sources. In 2008, Riahi et al. [9] used an artificial neural network system to differentiate between 


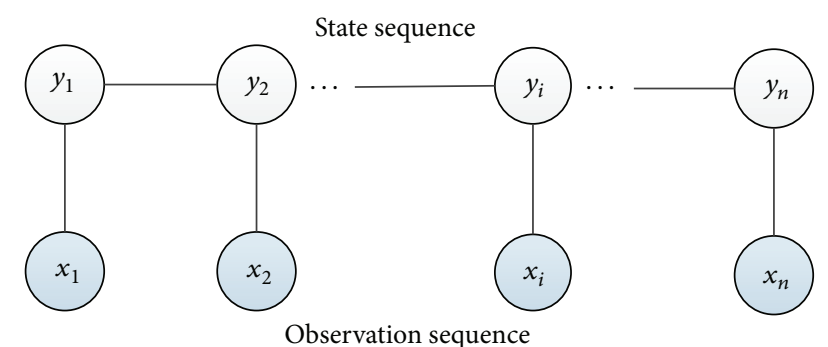

FIGURE 1: Structure of linear chain conditional random field.

leakage and corrosion signals in AE testing of aboveground storage tank floors. Zhang et al. [10] proposed a method to detect the leakage of the gas pipeline valve by using $\mathrm{AE}$ technique and SVM (Support Vector Machine) was applied to recognize the leak level of the valve accurately. And in the field of tool wear monitoring, Zhu et al. [11], Varma and Baras [12], Zhang et al. [13], and Chen et al. [14] both used HMM (Hidden Markov Model) to recognize the different tool wear states.

In this study, an algorithm based on CRF (Conditional Random Field) model is proposed to differentiate drop-back noise from corrosion AE signals. Seven typical AE parameters, such as amplitude, counts, duration time, rise time, true energy, average frequency, and peak frequency, are extracted to create the classifier model by CRF, BP (Back Propagation), SVM, and HMM. The result showed that CRF model is better than the other three models in training speed, accuracy, and ROC (Receiver Operating Characteristic) results.

This paper is organized as follows. Section 2 introduces the basic principles of CRF model. The experimental setup and procedure are illustrated and feature extraction method for the AE signals is presented in Section 3. Section 4 shows the establishment procedure of CRF model creating and the results, which are obtained by the comparison between CRF and other three classifiers. Section 5 presents results of application of CRF model in the field experiment. And the summary of the paper is given in Section 6.

\section{CRF Model}

CRF model is a typical discriminant model which was proposed by Lafferty et al. in 2001 [15]. A CRF may be viewed as an undirected graphical model, or Markov random field, which defines a single log-linear distribution over output variable sequences given a particular input random variable [16].

Linear chain conditional random field (LC-CRF), shown in Figure 1, is one of the most commonly used forms of the CRF model. The input random variable $X=$ $\left\{x_{1}, x_{2}, x_{3}, \ldots, x_{t}\right\}$ and the output random variable $Y=$ $\left\{y_{1}, y_{2}, y_{3}, \ldots, y_{t}\right\}$ denote the observation sequence and the state sequence, respectively. If the conditional probability of $Y$ given $X$ is known, $Y$ tends to satisfy the maximum global conditional probability $Y^{*}$; that is,

$$
Y^{*}=\arg \max _{Y} P(Y \mid X) .
$$

In this model, for the observation data $x$, the probability of the state sequence $y$ can be represented as

$$
\begin{aligned}
& P(y \mid x)=\frac{1}{Z(x)} \\
& \cdot \exp \left(\sum_{i, k} \lambda_{k} t_{k}\left(y_{i-1}, y_{i}, x, i\right)+\sum_{i, l} \mu_{l} s_{l}\left(y_{i}, x, i\right)\right) .
\end{aligned}
$$

$Z(x)$ is a normalization factor which can be described as

$$
\begin{aligned}
& Z(x) \\
& \quad=\sum_{y} \exp \left(\sum_{i, k} \lambda_{k} t_{k}\left(y_{i-1}, y_{i}, x, i\right)+\sum_{i, l} \mu_{l} s_{l}\left(y_{i}, x, i\right)\right),
\end{aligned}
$$

where $t_{k}\left(y_{i-1}, y_{i}, x, i\right)$ is a transition feature function of the entire observation sequence and the states at positions $i$ and $i-1$ in the state sequence; $s_{l}\left(y_{i}, x, i\right)$ is a state feature function of the state at position $i$ and the observation sequence; $\lambda_{k}$ and $\mu_{l}$, which needed to be estimated from training data, denote the weight values of the transition feature function and the state feature function respectively.

For the AE testing on tank floors, the features extracted from the AE signals can be viewed as the observation sequence and the signal types can be viewed as the state sequence. Then, the CRF model can be created and the signals can be classified.

\section{Experimental Preparation and Feature Extraction}

3.1. Experimental Setup. The experimental system consists of a water tank, the inner $\mathrm{AE}$ detector, and a specimen for corrosion experiments, shown in Figure 2. The water tank in Figure 3, with the dimension of $1.4 \mathrm{~m} \times 1.4 \mathrm{~m} \times 1.5 \mathrm{~m}$ (length $\times$ width $\times$ height), is used to simulate a storage tank in the laboratory. The inner AE detector, which is utilized to collect AE signals, includes AE sensors, the amplifier, the data acquisition system, and batteries (see Figure 4(a)) [17]. The detector can actuate itself to get close to the tank floor and collect AE signals, so it could weaken the interference caused by external disturbance and improve the signal-to-noise ratio (SNR) compared with the conventional AE testing method on tank floors. Four AE sensors are mounted in the holes on the bottom of the detector to collect AE signals. And the data acquisition system including processing circuits, the $\mathrm{AD}$ sampling card, and the PC104 computer is placed inside the shell to sample and save the collected signals.

The specimen, shown in Figure 4(b), is corroded by acid to simulate the corrosion in tank floor. The material of the specimen is the Q235 carbon steel sheet, which is identical with the material of the storage tank floors. The specimen is machined by the dimension of $180 \mathrm{~mm} \times 180 \mathrm{~mm} \times 5 \mathrm{~mm}$ (length $\times$ width $\times$ thickness) with the surface roughness of $0.02 \mathrm{~mm}$. A round, hollow vessel with the inner diameter of $50 \mathrm{~mm}$ is fixed on the specimen by epoxy. The surface of the specimen was grinded by abrasive papers through 400 -grade to 2000 -grade, rinsed with acetone, degreased with deionized 


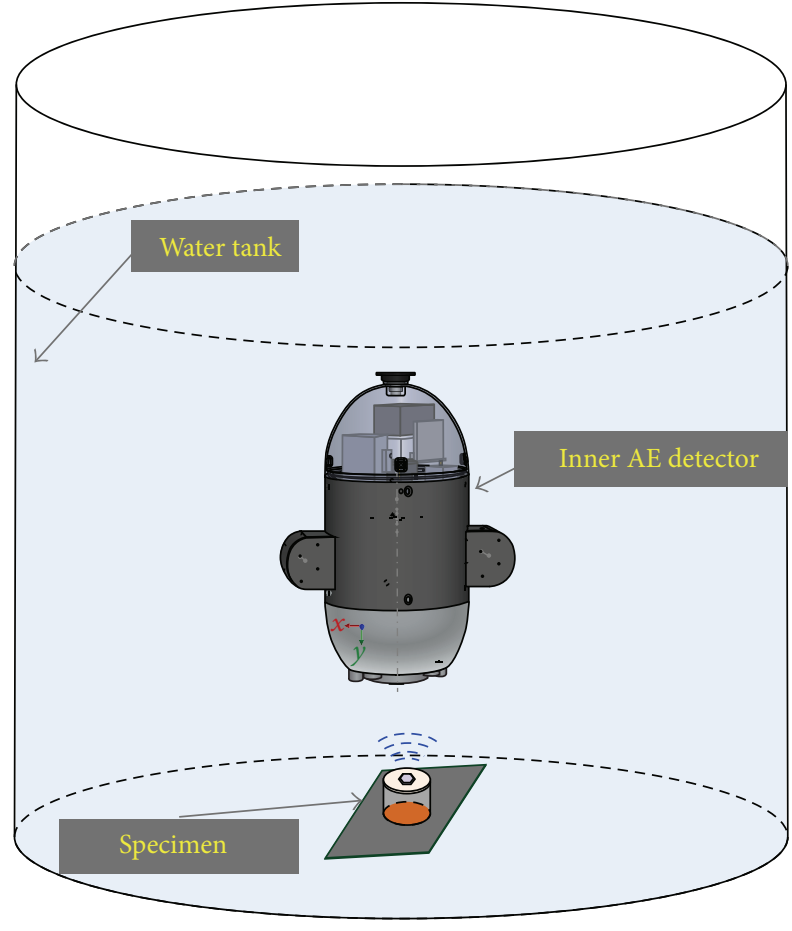

FIGURE 2: Diagram of the experimental system.

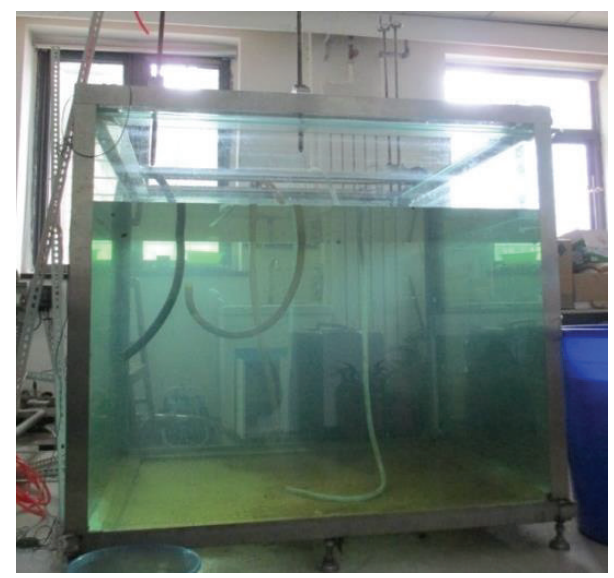

FIGURE 3: The water tank in the laboratory.

water, and dried in air. Before the experiments, the acid would be poured into the vessel and sealed with a lid wrapped with a matching ribbon.

\subsection{Experimental Procedure}

3.2.1. Collection of Corrosion Signals. To collect the corrosion signals, $5 \mathrm{~mol} / \mathrm{L} \mathrm{H}_{3} \mathrm{PO}_{4}$ was used as the test solution to react with the specimen to simulate the corrosion in tanks. R15 piezoelectric AE sensors produced by Physical Acoustics Corporation (PAC), with operating frequency range of 50$400 \mathrm{kHz}$, were used in the experiment. The gain of the charge preamplifier is set to $60 \mathrm{~dB}$, and the cut-off frequencies of the analog band pass filter are $100 \mathrm{kHz}$ and $400 \mathrm{kHz}[18,19]$.
TABLE 1: Seven typical characteristic parameters of corrosion AE signals.

\begin{tabular}{lcc}
\hline Hit based features & $\begin{array}{c}\text { Comprehensive } \\
\text { features }\end{array}$ & Frequency features \\
\hline $\begin{array}{l}\text { Amplitude } \\
\text { Counts }\end{array}$ & \\
$\begin{array}{l}\text { Duration time } \\
\text { Rise time }\end{array}$ & Average frequency & Peak frequency \\
True energy & & \\
\hline
\end{tabular}

The sampling rate is $3 \mathrm{MHz}$ and the sampling precision is 10-bit. During the experiment, the threshold level was fixed at $35 \mathrm{mV}$, which was slightly above the previously measured background noise.

A series of experiments were conducted in the laboratory. The specimen, which was handled in terms of the procedures mentioned before, was placed on the tank floor with a distance of $15 \mathrm{~cm}$ under the inner detector in the water tank and corrosion signals were collected for about 1 hour.

3.2.2. Collection of Drop-Back Signals. The field experiment was conducted in a new fire-resistant water tank in good condition. The diameter of the tank is $6 \mathrm{~m}$ and the height is $10 \mathrm{~m}$. The experiment preferences were the same as that in the lab test. The temperature during the experiment in the tank was $23^{\circ} \mathrm{C}$, while the outside temperature was $-15^{\circ} \mathrm{C}$. The drop-back signals were rich due to the difference between the warm gas in the tank and the fixed cold roof of the tank. After measuring the environment noise level, the threshold is set higher than the background noise. During the experiment, drop-back signals were collected without the eroded specimens (see Figures 5 and 6).

3.2.3. Collection of Mixed Signals. After collecting the dropback signals, in the field water tank, the eroded specimen was placed at the same position with the lab test. And both the corrosion signals and the interference signals were acquired. During the experiment of one hour, 7475 groups of AE signals were collected for further analysis.

3.3. AE Feature Extraction and Sample Set. The feature of AE parameters represents characteristics of the corrosion signals, and seven typical feature parameters of AE signals are extracted to build the classification model [20, 21]. The features consists of five hit based features, one comprehensive feature and one frequency feature, shown in Table 1.

In order to realize the classification by pattern recognition, 260 groups of corrosion signals and 260 groups of dropback interference signals were selected as samples to establish the classification model. The signals were randomly divided into 2 sets. 200 groups signals were used as the training set while the other 60 groups were used as test set, respectively. The formation of the training set and the test set are listed in Table 2.

3.4. Feature Selection. As stated, seven AE features, such as amplitude, counts, duration time, rise time, true energy, average frequency, and peak frequency, are extracted and 


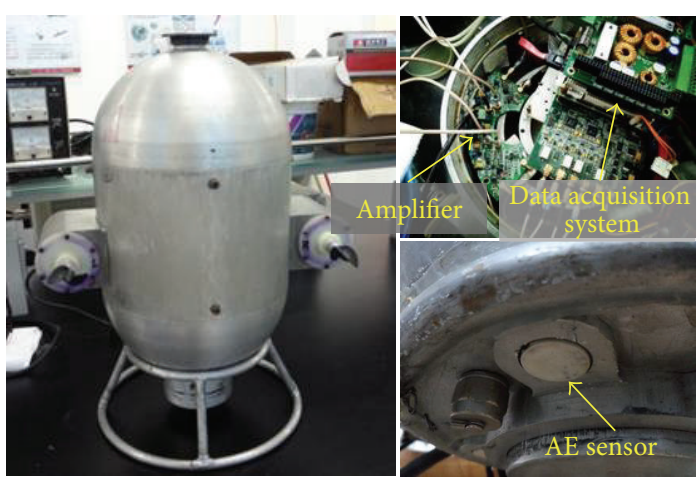

(a)

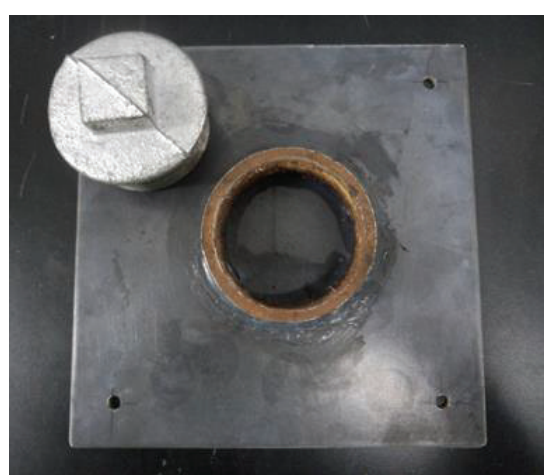

(b)

FIGURE 4: (a) Inner AE detector; (b) the specimen.

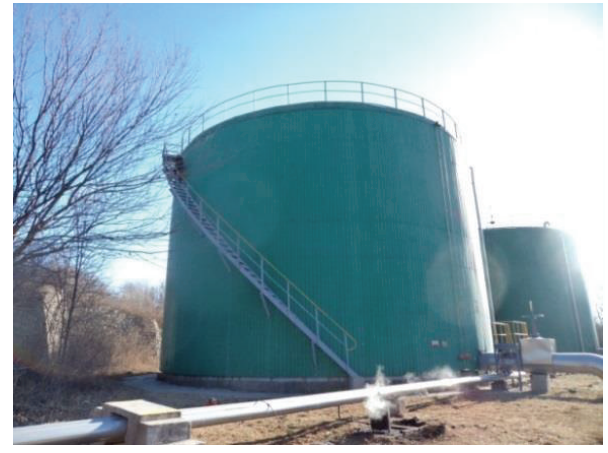

FIGURE 5: The fire-resistant water tank in field test.

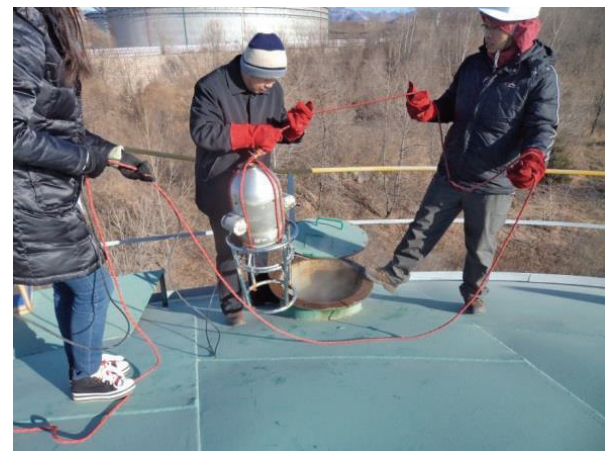

Figure 6: Testing in the field.

they are defined as F1 F7. To avoid the influence caused by the different magnitude between seven features, characteristic parameters are normalized to $[-1,1]$. And a feature selection algorithm named mRMR (Minimum Redundancy Maximum Relevance) is utilized to decide the optimal feature set.

mRMR is a new method to select good features proposed by Peng et al. [22]. It is going to find out the features with the highest relevance to the target class while still having
TABLE 2: Constitution of training set and test set.

\begin{tabular}{llc}
\hline Set name & Samples & Source of the signals \\
\hline \multirow{2}{*}{$\begin{array}{l}\text { Training } \\
\text { set }\end{array}$} & $\begin{array}{l}\text { 200 groups of corrosion signal } \\
\text { features } \\
200 \text { groups of drop-back } \\
\text { interference features }\end{array}$ & Laboratory test \\
\hline \multirow{3}{*}{ Test set } & $\begin{array}{l}\text { 60 groups of corrosion signal } \\
\text { features } \\
60 \text { groups of drop-back } \\
\text { interference features }\end{array}$ & Field test \\
\hline
\end{tabular}

low redundancy with other features. And mRMR could be defined as

$$
\begin{aligned}
& \max D(S, c), \quad D=\frac{1}{|S|^{2}} \sum_{x_{i} \in S} I\left(x_{i}, c\right), \\
& \min R(S), \quad R=\frac{1}{|S|^{2}} \sum_{x_{i}, x_{j} \in S} I\left(x_{i}, x_{j}\right),
\end{aligned}
$$

where $S$ is the initial feature set and $c$ is the target class set, $I\left(x_{i}, c\right)$ is the mutual information of feature $x_{i}$ and class $c, D$ is the mean value of all mutual information values between individual feature $x_{i}$ and class $c, I\left(x_{i}, x_{j}\right)$ is the mutual information of features $x_{i}$ and $x_{j}$, and $R$ means the mutual information between different features.

Mutual information is defined in terms of their probabilistic density functions, given two random variables $x$ and $y$ :

$$
I(x, y)=\iint p(x, y) \log \frac{p(x, y)}{p(x) p(y)} d x d y .
$$

The operator $\Phi(D, R)$ is defined to combine $D$ and $R$ and consider the following simplest form to optimize $D$ and $R$ :

$$
\max \Phi(D, R), \quad \Phi(D, R)=D-R .
$$

And the result of the formula (6) is called Mutual Information Difference (MID) and it is used to rank features. In practice, seven features are ranked by $\mathrm{mRMR}$ as follows: 
F7, F4, F1, F3, F2, F6, F5. The first 4 features (peak frequency, rise time, amplitude, and duration time) are determined as the optimal feature set to train and recognize the samples.

\section{Classification Results and Discussions}

In this section, the classifier models of CRF, BP, SVM, and HMM are adopted to recognize the corrosion signals from interference signals based on the extracted features, respectively. And the results of the different models are compared and discussed.

4.1. Establishment of CRF Classification Model. In the LCCRF model, the feature vectors of input sequences are considered to be positive integers. And thus, the extracted features are normalized to 1 101 and used as the observation sequences. The state of the sample for corrosions and dropback interferences is labeled as 1 and 2, respectively. The application of the LC-CRF model includes two steps: training and recognition. The features of the training samples are used to calculate the model parameters $(\lambda, \mu)$. The conditional probability model is obtained by means of the maximum likelihood estimation, while the limited-memory BroydenFletcher-Goldfarb-Shanno (LBFGS) algorithm is used to get the optimal parameters for the model. Here, the initial model parameters are set to 0 and the convergent accuracy limit is 0.0001 . During recognition, features of the test samples are taken as input variables while the state sequences for the model, obtained in the first step, are calculated by the Viterbi algorithm. The method can be summarized by the flowchart illustrated in Figure 7.

4.2. Establishment of BP, SVM, and HMM. As stated, BP, SVM, and HMM are commonly used methods for classification of $\mathrm{AE}$ signals. In order to compare with the recognition results, the three classifiers use the same training and test data as LC-CRF. The models of BP, SVM, and HMM are established as follows.

BP is a common method of training artificial neural networks. The structure of a typical BP classifier is shown in Figure 8. To design a BP model, these parameters should be determined: function of the output layer, function of the hidden layer, training rate, and the number of hidden layer nodes. In the tests, " $S$ " function is selected as the activation function of the hidden layer, and " $P$ " function is chosen as the transfer function of the output layer. And the learning values are tuned by a gradient descent manner. The number of hidden layer nodes, set as 14 in this test, was twice the number of input nodes. In addition, learning rate is 0.01 .

The SVM uses the central concept named kernel for a number of tasks. Kernel machines provide a modular framework that can be adapted to different tasks and domains by using different kernel function and base algorithm. The structure of SVM is shown in Figure 9, where $k$ is the kernel function. Three parameters need to be determined to design a SVM model: the kernel function, the cost $(c)$, and the gamma $(g)$. In this paper, the classical RBF kernel function is chosen, where kernel parameters $g$ and $c$ could be determined
TABLE 3: Classification results using different features.

\begin{tabular}{lc}
\hline Feature set & Accuracy rate (\%) \\
\hline F1 F7 & 99.17 \\
F7, F4, F1, F3, F2, & 100 \\
F7, F4, F1, F3, & 100 \\
F7, F4, F1 & $99.17 \%$ \\
\hline
\end{tabular}

TABLE 4: Classification results.

\begin{tabular}{lcc}
\hline Method & Max training time (s) & Accuracy rate (\%) \\
\hline BP & 9.62 & 90.0 \\
SVM & 3.97 & 97.5 \\
CRF & 1.03 & 100 \\
HMM & 3.68 & 83.3333 \\
\hline
\end{tabular}

by fivefold cross-validation methodology. In that case, the optimal solution $c$ is 2 , and $g$ is 22.627 .

HMM is composed of Markov chain and stochastic process. The Markov chain corresponds to the state sequence, which is described by $\pi$ and $A$. The stochastic process is depicted as the observation sequence, which is described by $B$. So a HMM model can be described as

$$
\lambda=(M, N, A, B, \pi),
$$

where $M$ is the state number of the Markov chain and $N$ is the possible number of observed value in each state. $A$ is state transfer probability matrix with sizes $N \times N$ and $B$ is the probability matrix of the observed values whose sizes are equal to $N \times M . \pi$ is the initial probability distribution vector with length $N$. So $M$ and $N$ must be confirmed to establish a HMM classifier. The value of $M$ and $N$ is decided as 6 and 8 , respectively, using the trial and error method. The model parameters are calculated by Baum-Welch algorithm, while the convergent accuracy limit is 0.0001 .

4.3. Results and Discussions. To validate that if the first 4 features are the optimal feature set, samples are trained and tested by CRF model using the first 3 , the first 5 , and all seven features, respectively. The accuracy rates are shown in Table 3. Using the first 4 and the first 5 features can make an accuracy rate of $100 \%$, higher than the other two feature sets. So the first 4 features (peak frequency, rise time, amplitude, and duration time) are selected as the optimal feature set to train and recognize the samples.

Using same training and test set, the recognition results of CRF model and the other three algorithms (BP, SVM, and HMM) are compared based on a PC (Core 2 Duo E6300 with $3.2 \mathrm{G}$ memory), respectively. The results are compared in the training time, the accuracy, and the ROC (Receiver Operating Characteristic) curve. The maximum training time and accuracy rate are shown in Table 4.

It shows that the accuracy rate of the CRF is higher than BP, SVM, and HMM model and the training time of CRF is the shortest. Gradient descent algorithm is utilized to adjust the parameters of BP, so it needs to iterate to get the optimal parameters. Moreover, the selection of the maximum 


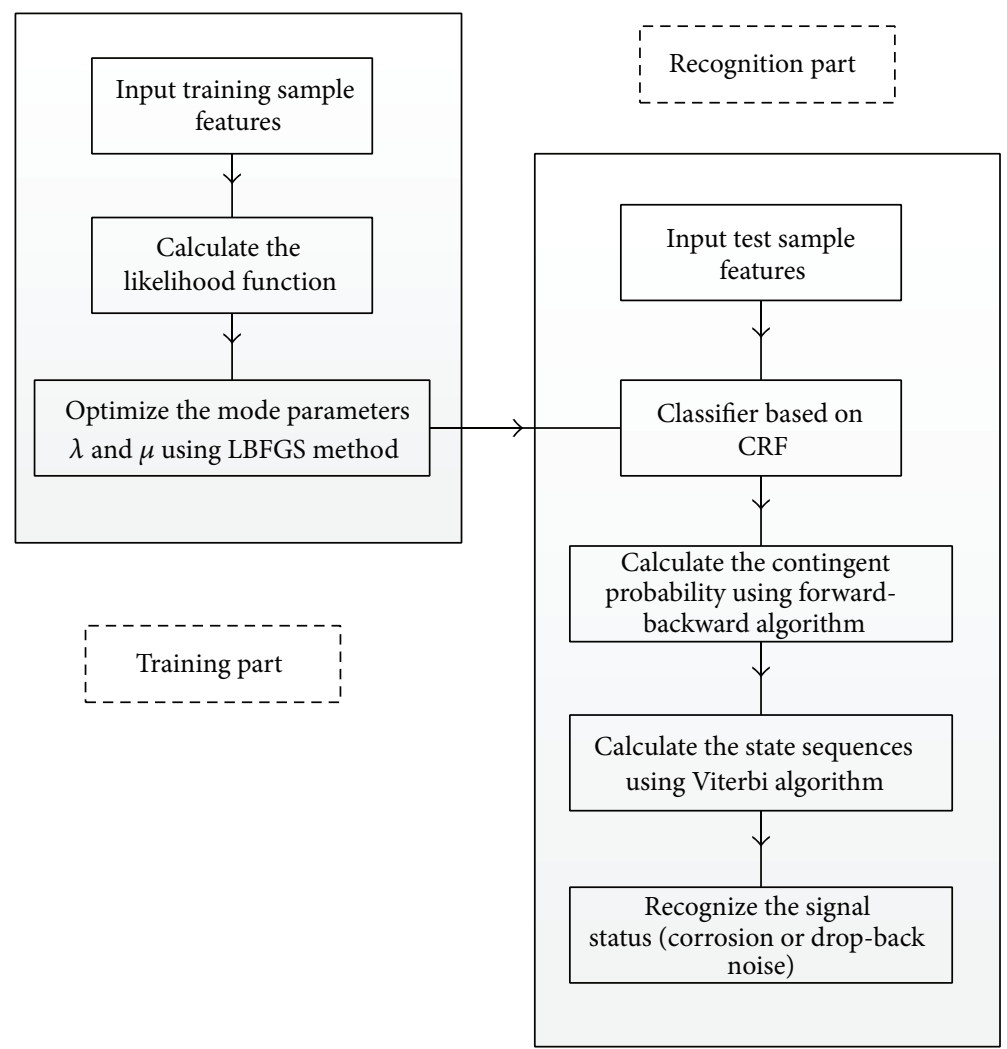

FIgURE 7: Flowchart of the identification process based on CRF.

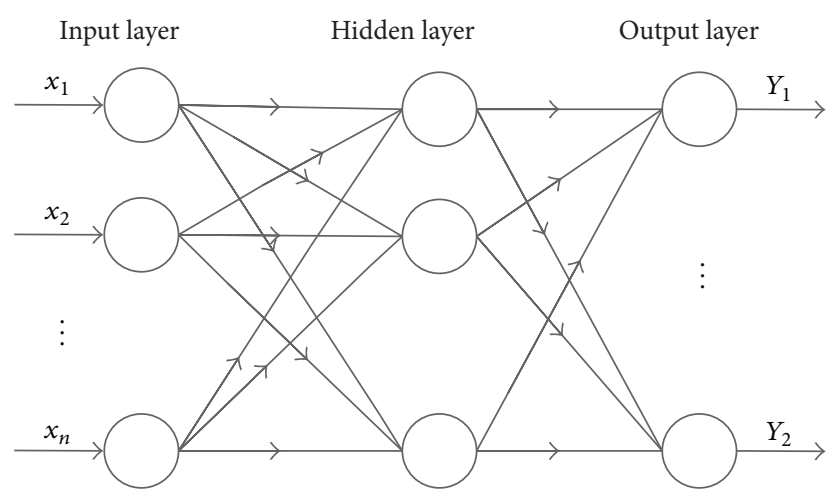

Figure 8: The architecture of the BP.

iteration, learning rate, and number of the hidden layer nodes are often determined by experience or method of trial and error. So the training speed and the accuracy rate of BP are lower and it is difficult to get the optimum network. The training speed and accuracy of SVM are higher than BP and HMM but it is more suitable for the situation of small sample data rather than $\mathrm{AE}$ testing field, which is a large sample data situation. HMM is widely employed in many fields, but one of the disadvantages of HMM is that this model assumes that the observation value at one point is only dependent on the state of Markov chain at this time and the observation sequences are independent of each other, while the features of

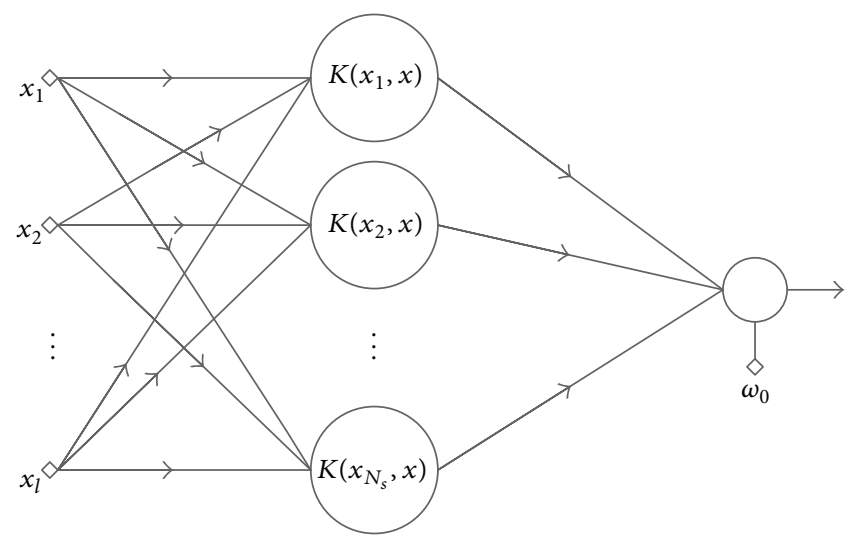

FIGURE 9: SVM architecture.

AE signals are not independent of each other. So the accuracy of HMM is the lowest in the four models. The CRF model could fully utilize the information of the features and accept the dependences between the features. Meanwhile, it could always achieve global optimization.

Furthermore, ROC curve is used to test the performance of CRF model and BP, SVM, and HMM model [23-25]. The curve is created by plotting the true positive rate (sensitivity) against the false positive rate $(1-$ specificity) at various threshold settings. The area under the curve (AUC) can be used as judge criteria of models. Larger AUC represents better 


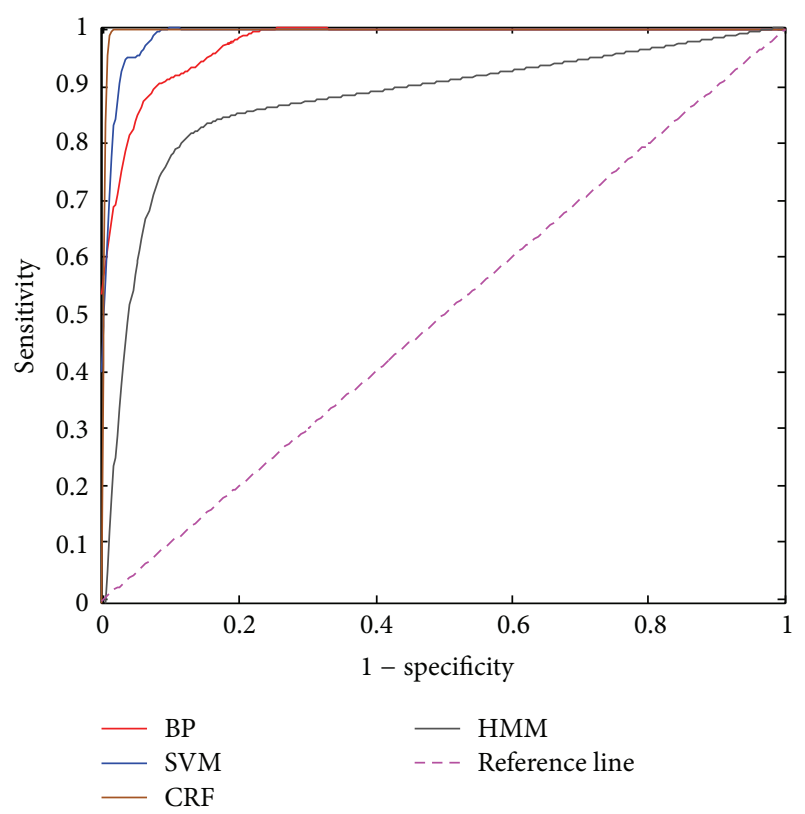

FIGURE 10: The ROC curve of the recognition results for corrosion signals.

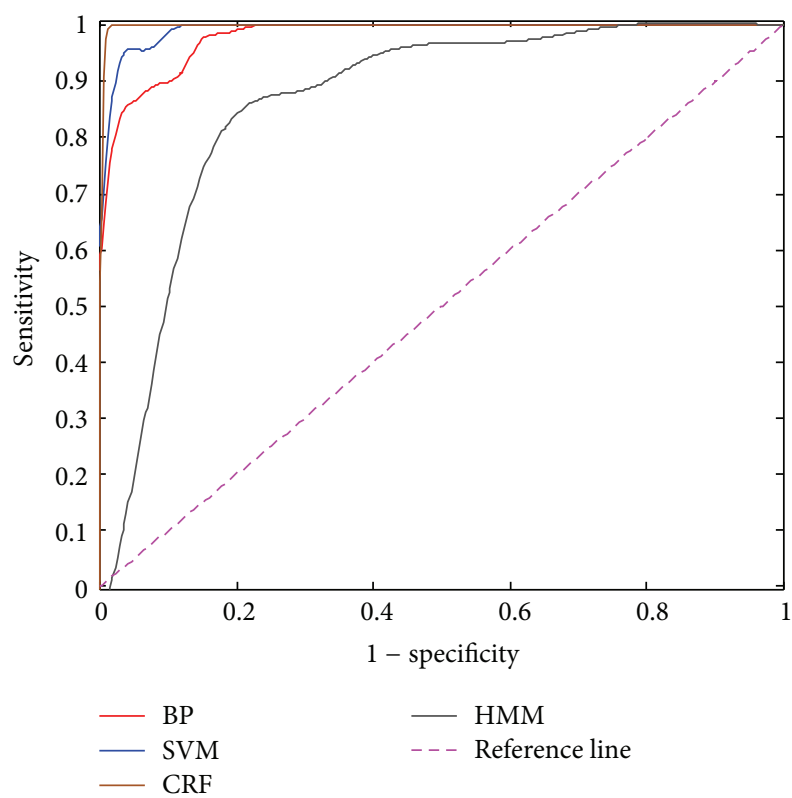

FIGURE 11: The ROC curve of the recognition results for drop-back interferences.

performance. Figures 9 and 10 show the ROC curves of the recognition results for corrosion signals and drop-back noises, respectively.

It is shown in Figures 10 and 11 that CRF model has the greatest AUC, followed by SVM, BP, and HMM model.

Therefore, the recognition result of CRF model outperforms SVM, BP, and HMM in training speed, accuracy rate, and the AUC of the ROC curves.

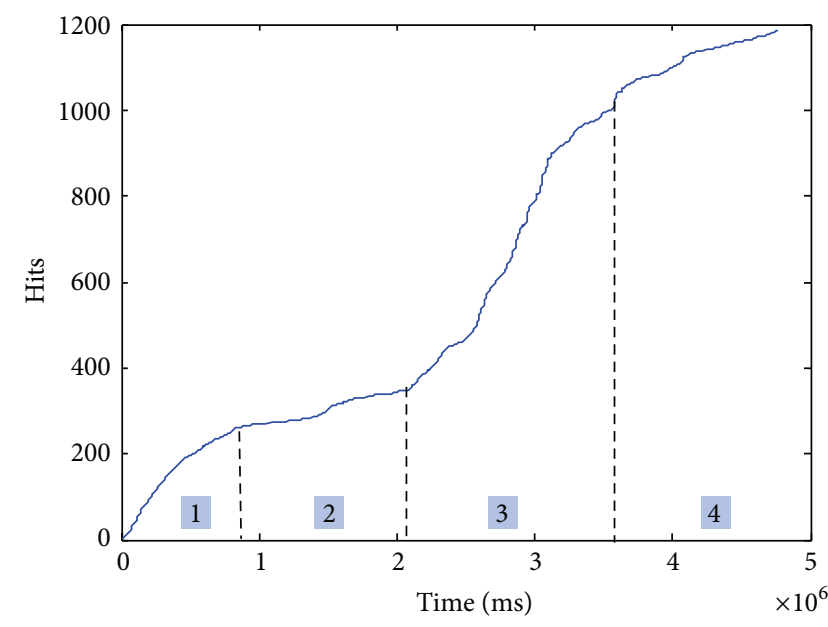

FIGURE 12: The relation curve of AE hits varying with time in laboratory test.

\section{Application of CRF Model in Field Experiments}

In the last step of field experiment, the inner detector collected both corrosion signals and drop-back noise. During the experiment of one hour, 7475 groups of AE signals were collected and classified using CRF model. 1105 groups were classified as corrosion signals and the other 6370 groups were identified as drop-back interferences. And the quantity of corrosion signals is approximately equal to the quantity of corrosion signals collected in the laboratory in the same duration. To test the effect of CRF model in the field environment, statistical analysis method was used to compare the results. The relation curves, in which the cumulative quantity of signals varies with time, were obtained and showed in Figures 12 and 13.

In Figure 12, the corrosion signals were collected in the laboratory and the quantity of AE hits (corrosion signals) varies with time. It is observed that the corrosion process can be divided into 4 zones. At the beginning (Zone 1), the phosphoric acid began to react with the steel plate. Because of the large contact area and high hydrogen ion concentration, the quantity of $\mathrm{AE}$ hits increases fast. Then, the hydrogen created during the reaction accumulated on the surface of the plate and formed bubbles so that the contact area was decreased (Zone 2). As the reaction progressed, the bubbles converged into large bubbles and then burst out. The acid was fully contacted with the steel plate again and the reaction rate and the growth rate of $\mathrm{AE}$ hits increased dramatically (Zone 3). While the concentration of hydrogen ion fell, the acid reacted with the steel plate slower than before and the quantity of AE hits grew slowly (Zone 4).

Figure 13(a) shows the relation between the quantity of $\mathrm{AE}$ hits and time before being classified by CRF model and it is almost linear. It does not reflect the statistical law of the corrosion tests. Figure 13(b) shows the relation curve of $\mathrm{AE}$ hits and time of the signals collected in field site after classified by CRF model. The relation curve in Figure 13(b) 


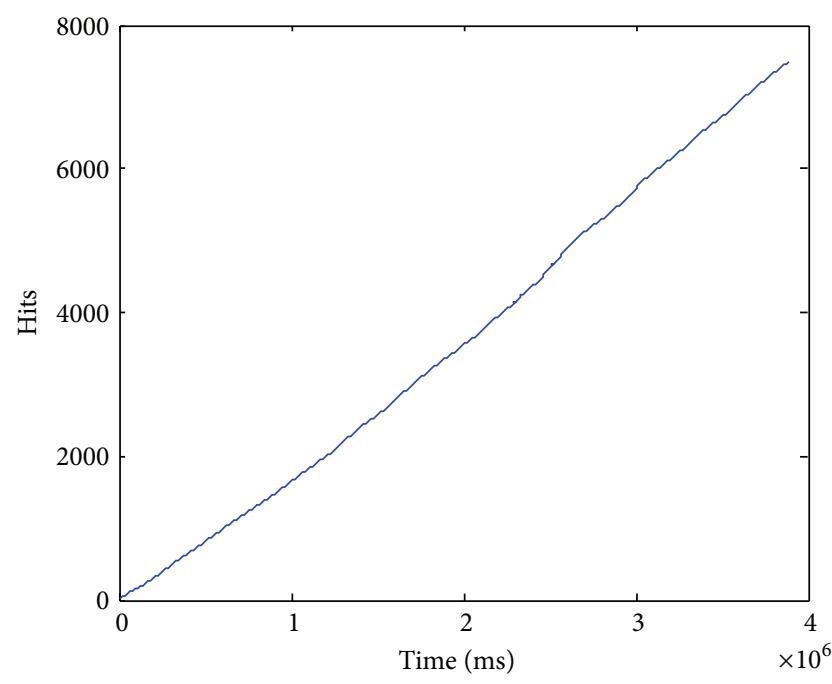

(a)

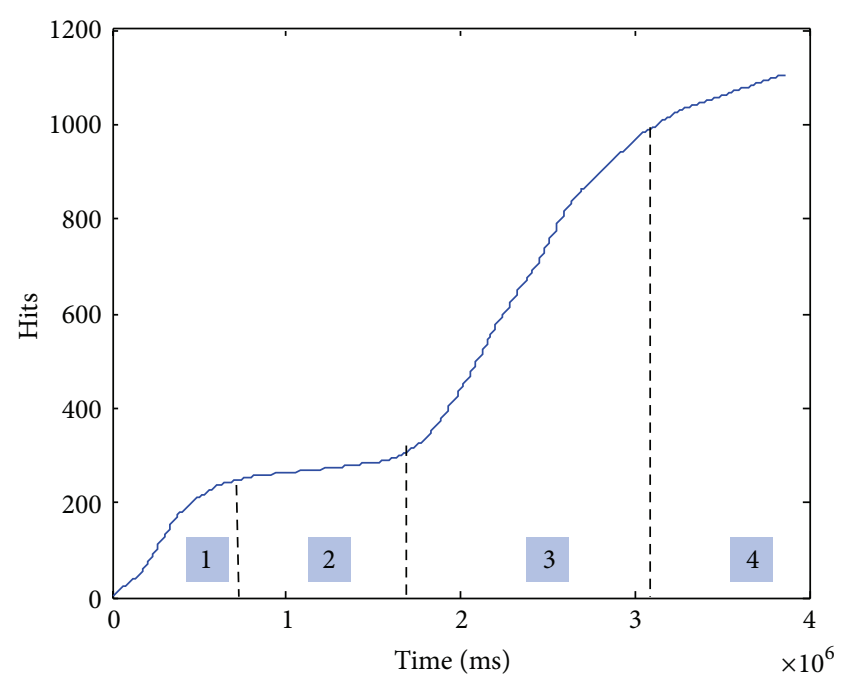

(b)

FIGURE 13: The relation curve of AE hits varying with time in field test. (a) Signals unclassified by CRF. (b) Signals classified by CRF.

also has 4 zones with same characteristics in Figure 12. There is a subtle difference between turning point of the zones on time axis in Figure 13(b) and that in Figure 12 because the setup time for inner detector to start collecting signals in the field test was a litter longer than in the laboratory test. The result shows that the data processed using CRF model could reflect the statistical law of the corrosion test and the CRF model performs well in the field test application.

\section{Conclusions}

Drop-back signals which are caused by condensation in storage tanks with fixed roofs are a big problem in AE online storage tank floor inspection. In this paper, a new inner $\mathrm{AE}$ detector and a recognition algorithm based on CRF model are applied to differentiate corrosion $\mathrm{AE}$ signals from drop-back interferences. AE parameters, amplitude, counts, duration time, rise time, true energy, average frequency, and peak frequency, were selected as feature parameters for recognition.

Experiments were carried out in water tanks both in laboratory and in the field to collect corrosion AE signals and drop-back signals. The recognition results of CRF are compared with other 3 models of BP, SVM, and HMM. The comparisons of the accuracy, training speed, and the AUC of the ROC curve show that the CRF outperforms the other three models for the recognition of corrosion signals and drop-back interference signals.

\section{Conflict of Interests}

The authors declare that there is no conflict of interests regarding the publication of this paper.

\section{Acknowledgments}

This work is supported by Tianjin Science Foundation under the Grant of 13JCYBJC18000, Tianjin Technical-Support
Foundation under the Grant of 14ZCZDGX00003, and Tianjin Marine Economy Innovation and Development Region Demonstration Project under the Grant of 2015120024000473.

\section{References}

[1] M. C. Lin, Y. W. Kang, W. B. Wang, L. Zhang, and Y. Sun, "Research on acoustic emission in-service inspection for large above-ground storage tank floors," in Proceedings of the 8th International Pipeline Conference (IPC '10), pp. 233-239, Calgary, Canada, October 2010.

[2] L. Y. Sun and Y. B. Li, "Large vertical storage tank bottom evaluation via acoustic emission signal analysis," in Proceedings of the 23rd Chinese Control and Decision Conference (CCDC '11), pp. 3554-3558, Mianyang, China, May 2011.

[3] S. Park, S. Kitsukawa, K. Katoh, S. Yuyama, H. Maruyama, and K. Sekine, "Development of AE monitoring method for corrosion damage of the bottom plate in oil storage tank on the neutral sand under loading," Materials Transactions, vol. 47, no. 4, pp. 1240-1246, 2006.

[4] M. Sanchez, N. Cardenas, and V. A. Dominguez, "Acoustic emission testing of aboveground petroleum storage tanks: risk assessment and lessons learned," Process Safety Progress, vol. 31, no. 2, pp. 159-164, 2012.

[5] W.-K. Wang, Y.-B. Li, Y.-N. Li, and Y. Zhang, "An Acoustic emission event determination method for acoustic emission testing of tank bottom based on cluster analysis," in Proceedings of the IEEE/ASME International Conference on Advanced Intelligent Mechatronics: Mechatronics for Human Wellbeing (AIM '13), pp. 1686-1691, Wollongong, Australia, July 2013.

[6] Y. H. Huang, H. L. Chong, M. Hu, X. M. Jia, W. Liu, and J. Ma, "Application and research of acoustic emission technology in the tank security detection," Key Engineering Materials, vol. 501, pp. 561-565, 2012.

[7] T. L. Fu, Studies on key technologies in tank bottom corrosion internal detection system [M.S. dissertation], Tianjin University, Tianjin, China, 2012. 
[8] D. Gang, J. Shijiu, Z. Congying, and W. Weikui, "A study on acoustic emission technology for tank bottom corrosion inspection," Anti-Corrosion Methods and Materials, vol. 57, no. 6, pp. 275-279, 2010.

[9] M. Riahi, H. Shamekh, and B. Khosrowzadeh, "Differentiation of leakage and corrosion signals in acoustic emission testing of aboveground storage tank floors with artificial neural networks," Russian Journal of Nondestructive Testing, vol. 44, no. 6, pp. 436-441, 2008.

[10] H. F. Zhang, Z. L. Li, Z. L. Ji, and Z. Bi, "Intelligent leak level recognition of gas pipeline valve using wavelet packet energy and support vector machine model," Insight, vol. 55, no. 12, pp. 670-674, 2013.

[11] K. P. Zhu, G. S. Hong, and Y. S. Wong, "A comparative study of feature selection for hidden Markov model-based micromilling tool wear monitoring," Machining Science and Technology, vol. 12, no. 3, pp. 348-369, 2008.

[12] S. Varma and J. S. Baras, "Tool wear estimation from acoustic emissions: a model incorporating wear-rate," in Proceedings of the IEEE 16th International Conference on Pattern Recognition, pp. 492-495, Quebec City, Canada, 2002.

[13] X. Zhang, H.-Y. Fu, Y.-Z. Sun, and Z.-Y. Han, "Hidden Markov model based micro-milling tool wear monitoring," Computer Integrated Manufacturing Systems, vol. 18, no. 1, pp. 141-148, 2012.

[14] Y. L. Chen, Z. F. Tian, and D. Z. Hou, "The acoustic emission monitoring method of tool wear and breakage in CNC boring," Machine Tool \& Hydraulics, vol. 40, pp. 111-113, 2012.

[15] J. D. Lafferty, A. MeCallum, and F. C. N. Pereira, "Conditional random fields: probabilistic models for segmenting and labeling sequence data," in Proceedings of the 18th International Conference on Machine Learning (ICML '01), pp. 282-289, Williamstown, Mass, USA, 2001.

[16] H. M. Wallach, "Conditional random fields: an introduction," Technical Reports (CIS), 2004.

[17] T. L. Fu, Y. B. Li, and S. J. Jin, "Acquisition system of storage tank bottom corrosion acoustic emission based on PC104," Transducer and Microsystem Technologies, vol. 31, pp. 134-136, 2012.

[18] G. Du, W. Wang, S. Song, and S. Jin, "Detection of corrosion on 304 stainless steel by acoustic emission measurement," AntiCorrosion Methods and Materials, vol. 57, no. 3, pp. 126-132, 2010.

[19] J. Li, Nondestructive Testing Handbook, China Machine Press, Beijing, China, 2nd edition, 2011.

[20] P. O. Moore, Nondestructive Testing Handbook: Acoustic Emission Testing, American Society for Nondestructive Testing, Columbus, Ohio, USA, 3rd edition, 2005.

[21] L. Gao, F. Zai, S. Su, H. Wang, P. Chen, and L. Liu, "Study and application of acoustic emission testing in fault diagnosis of low-speed heavy-duty gears," Sensors, vol. 11, no. 1, pp. 599-611, 2011.

[22] H. Peng, C. Ding, and F. Long, "Minimum redundancy maximum relevance feature selection," IEEE Intelligent Systems, vol. 20, pp. 70-71, 2005.

[23] T. W. Yu, "ROCS: receiver operating characteristic surface for class-skewed high-throughput data," PLOS ONE, vol. 7, no. 7, Article ID e40598, 2012.

[24] D. D. Dorfman and K. S. Berbaum, "A contaminated binormal model for ROC data-Part III. Initial evaluation with detection ROC data," Academic Radiology, vol. 7, no. 6, pp. 438-447, 2000.

[25] J. Han, M. Kamber, and J. Pei, Data Mining: Concepts and Techniques, China Machine Press, 3rd edition, 2012. 

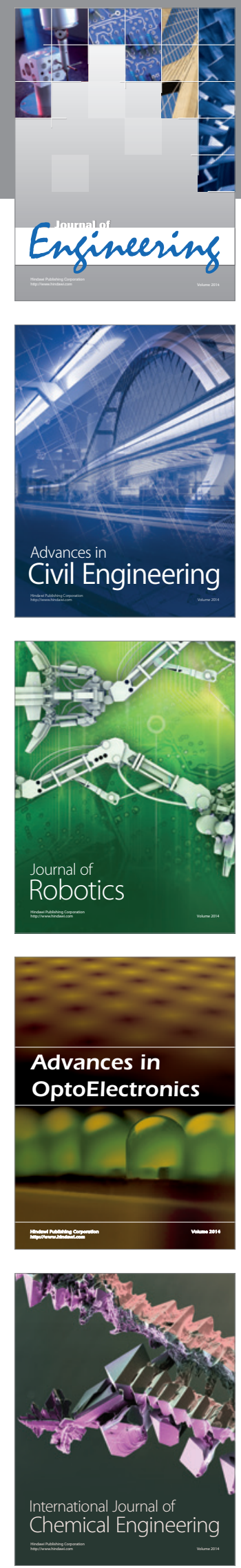

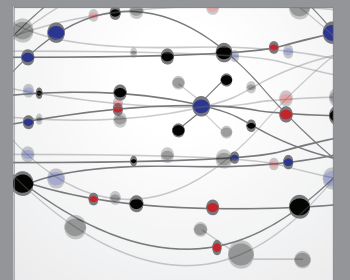

The Scientific World Journal
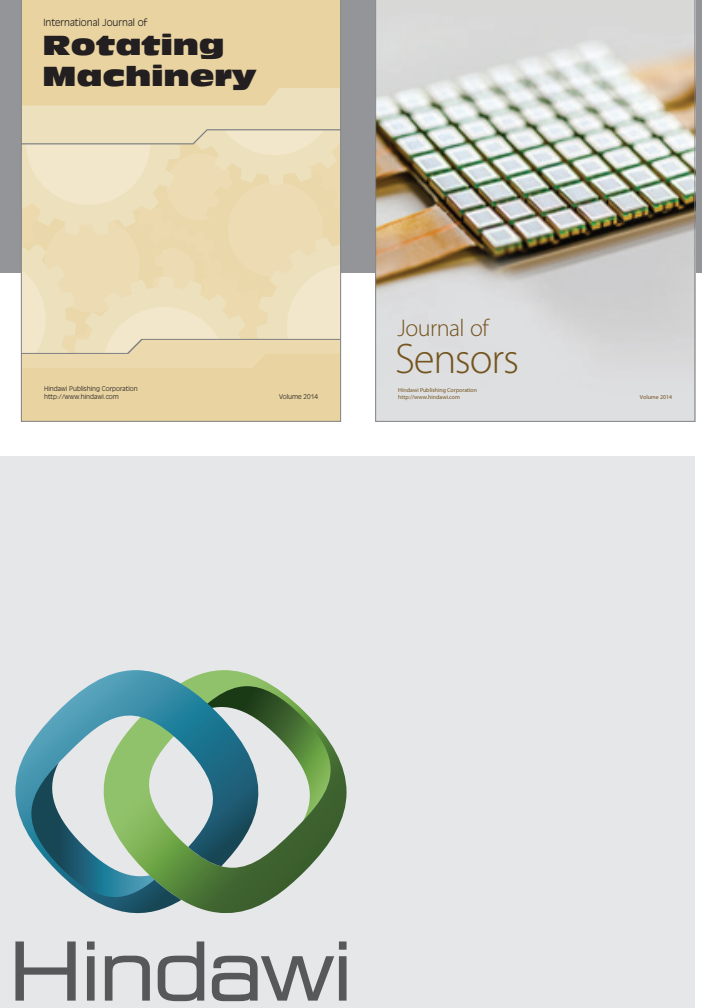

Submit your manuscripts at http://www.hindawi.com
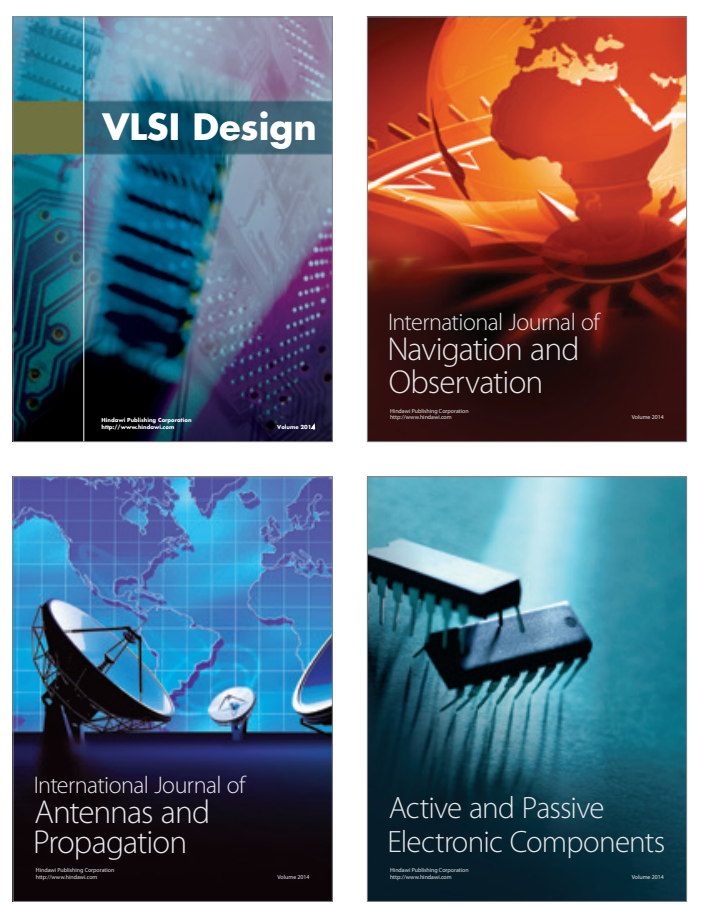
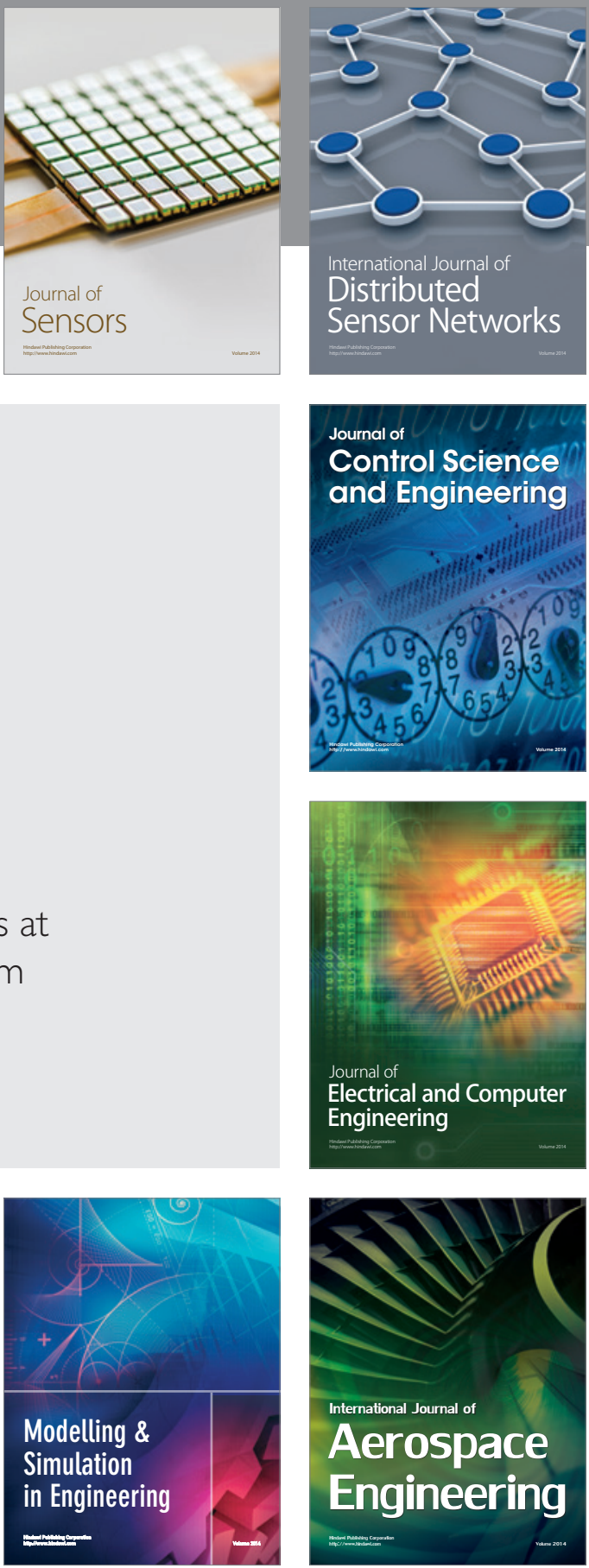

Journal of

Control Science

and Engineering
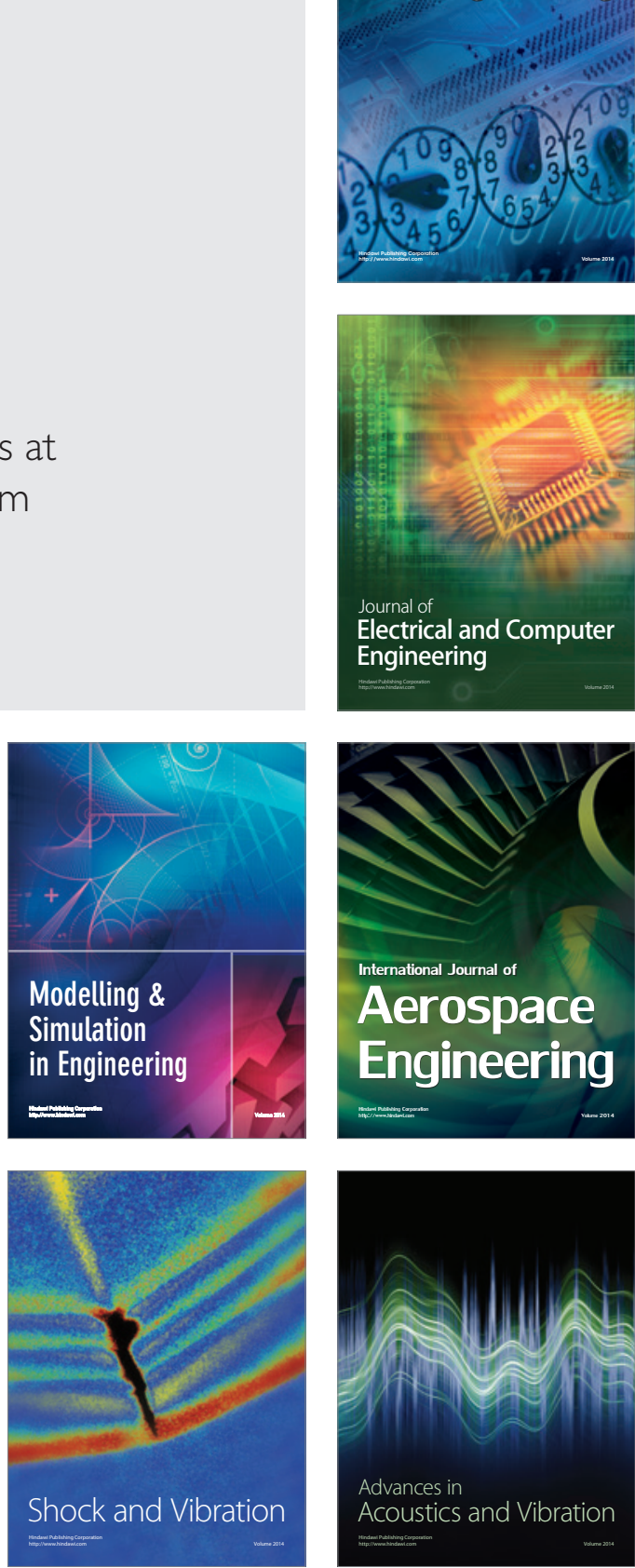